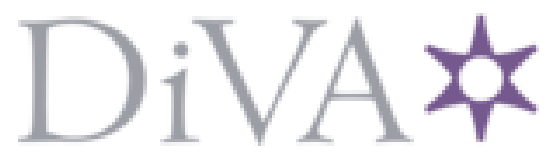

http://www.diva-portal.org

This is the published version of a paper published in Clinical Child Psychology and Psychiatry.

Citation for the original published paper (version of record):

Persson, S., Hagquist, C., Michelson, D. (2017)

Young voices in mental health care: Exploring children's and adolescents' service experiences and preferences.

Clinical Child Psychology and Psychiatry, 22(1): 140-151

https://doi.org/10.1177/1359104516656722

Access to the published version may require subscription.

N.B. When citing this work, cite the original published paper.

Permanent link to this version:

http://urn.kb.se/resolve?urn=urn:nbn:se:kau:diva-47611 


\title{
Young voices in mental health care: Exploring children's and adolescents' service experiences and preferences
}

\section{Stefan Persson', Curt Hagquist' and Daniel Michelson ${ }^{2,3}$}

'Centre for Research on Child and Adolescent Mental Health, Karlstad University, Sweden 2Department of Psychology, Institute of Psychiatry, Psychology \& Neuroscience, King's College London, UK

${ }^{3}$ Centre for Evidence-Based Intervention, Department of Social Policy and Intervention, University of Oxford, UK

\begin{abstract}
The development of 'youth-friendly' services has become a priority across a wide range of healthcare contexts. However, relatively few studies have specifically examined users' experiences of, and preferences for, child and adolescent mental health care. The current study investigated young service users' views of outpatient and community mental health clinics in Sweden, based on two data sources. First, focus group interviews were conducted with seven children and adolescents (aged 10-18 years) to explore both positive and negative experiences of mental health care. Second, written suggestions about specific service improvements were obtained from 106 children and adolescents. Qualitative content analysis revealed three overarching themes: 'Accessibility', 'Being heard and seen' and 'Usefulness of sessions'. Young people's recommendations for improving practice included more convenient appointment times, offered in welcoming settings; opportunities to communicate more openly with clinical staff, enabling sensitive discussion of mental health and wider personal issues; and more structured treatments that offer greater credibility and relevance to young people's mental health and developmental needs. Young people also discussed being compelled by parents and school professionals to engage in treatment. Attending to young people's preferences must be a priority in order to overcome ambivalence about session attendance, and enhance treatment participation and outcomes.
\end{abstract}

\section{Keywords}

Service user experience, outpatient, qualitative, mental health, child and adolescent mental health services

\section{Corresponding author:}

Stefan Persson, Centre for Research on Child and Adolescent Mental Health, Karlstad University, SE-65I 88 Karlstad, Sweden.

Email: stefan.persson@kau.se 


\section{Introduction}

Since the 1980s, upward trends in youth mental health problems have been observed across a number of countries (e.g. Bor, Dean, Najman, \& Hayatbakhsh, 2014). Simultaneously, increasing emphasis has been placed on mental health services being more attuned to the needs of young people as consumers and stakeholders (World Health Organization (WHO), 2012). In Sweden, for example, the number of children, adolescents and young adults (aged 0-24 years) receiving help at specialist psychiatric services has increased by one-third since 2000 (National Board of Health and Welfare, 2013). Nevertheless, the majority of youth with mental health needs remain untreated or undertreated. In a community sample of adolescents, only one-third of those above the 99th percentile for severity of emotional problems had sought help for their mental health (Zachrisson, Rödje, \& Mykletun, 2006). Furthermore, international research suggests that up to $75 \%$ of presenting cases do not complete a full course of treatment in child and adolescent mental health services (CAMHS) (for review, see de Haan, Boon, de Jong, Hoeve, \& Vermeiren, 2013).

The context for help-seeking has important implications for understanding youth service engagement and outcomes. Referrals to CAMHS are typically initiated by parents, teachers or other influential adults in the child's network (Ivert et al., 2011). In contrast, most adults seek out mental health care themselves. Being externally motivated to engage in treatment has been associated with resistance to change, whereas intrinsic motivation promotes more positive clinical outcomes (see Ryan \& Deci, 2008). Furthermore, previous research has found poor agreement between parents and children on the reasons for help-seeking and goals for treatment (Garland, Lewczyk Boxmeyer, Gabayan, \& Hawley, 2004; Yeh \& Weisz, 2001). Parents and their children may also disagree on their respective experiences of services: child- and parent-reported satisfaction with mental health services are at best only moderately associated (Day, Michelson, \& Hassan, 2011; Garland, Haine, \& Lewczyk Boxmeyer, 2007). This underscores the importance of evaluating services from the distinctive perspective of young people, and not merely relying on proxy reports from parents and other adult informants (Day et al., 2011; McLeod, 2011).

To date, relatively few studies have directly investigated the experience of mental health care for young people. Our own scoping review identified only six studies that focused specifically on young people's views of outpatient and community mental health services, including four British studies (Buston, 2002; Day, Carey, \& Surgenor, 2006; Harper, Dickson, \& Bramwell, 2014; Strickland-Clark, Campbell, \& Dallos, 2000), one Swedish study (Hartzell, Seikkula, \& von Knorring, 2009) and one study conducted in Australia (McCann \& Lubman, 2012). Consistent with the wider literature on service experience in youth populations (Freake, Barley, \& Kent, 2007; Lavis \& Hewson, 2011), the majority of themes in these studies revolved around relational aspects of care, with the experience of being listened to, understood and taken seriously being key concerns for both children and adolescents. Parental involvement also emerged as a central theme. Adolescents reported that parents, when present, often set the agenda for what is discussed in sessions (Harper et al., 2014). Additional concerns were expressed about parents' potentially negative reactions to in-session disclosures (Day et al., 2006; Strickland-Clark et al., 2000). In terms of session content, children and adolescents typically wanted concrete advice and information about how to resolve or cope with their problems (Buston, 2002; Day et al., 2006; McCann \& Lubman, 2012). Structural aspects of treatment were also important, such that younger children (Day et al., 2006) asked for appointment times that did not interfere with school or leisure activities. Adolescents preferred to see the same person, and also endorsed shorter waiting times (Buston, 2002; Harper et al., 2014). The clinic environment did not emerge as a major concern, whereas this appears to be a more salient issue for young people in inpatient settings (Biering, 2010; Buston, 2002). 
This study aimed to extend the emerging literature on mental health-care experience for young people, using a unique combination of (1) exploratory focus groups with young service users (aged 10-18 years), and (2) youth-completed surveys focused on specific service improvements. The objectives were to (1) elaborate and contextualise international findings on young people's experiences of mental health care in a Swedish sample, and (2) identify ways in which young people's service preferences can be translated into practice.

\section{Method}

\section{Design}

The design involved two phases. In Phase I, semi-structured focus group interviews were conducted with children and adolescents in outpatient CAMHS, in order to explore their experiences of care. In Phase II, a combined sample of children and adolescents in outpatient and community clinics provided written suggestions about service improvements. The study was reviewed and approved by the Regional Ethical Review Board in Uppsala, Sweden (Dnr 2011/460).

\section{Study setting}

Phase I participants were recruited from a single CAMHS outpatient clinic serving youth aged 0-18 years in a midsized Swedish city ( $~ 50,000$ inhabitants). Participants in Phase II were recruited from 11 outpatient CAMHS and three community-based 'första linjen' ('first-line') mental health services situated in nine county regions across Sweden. The latter is comparable to 'Tier II' provision in the UK system (i.e. primary mental health workers delivering relatively low-intensity interventions for mild to moderate problems), whereas the former is similar to the conventional 'Tier III' model (i.e. multidisciplinary teams providing a specialised service for children and young people with more severe, complex and persistent problems).

\section{Participants}

Phase I. Inclusion criteria were (1) aged 10-18 years, (2) participation in at least 2 outpatient sessions of an individual or family-based psychological intervention at a specialist CAMHS clinic in the previous year, (3) Swedish speaking and (4) ability to provide informed consent. A total of 10 service users were approached, of which 7 participated in the study (one participant only took part in the validation stage). Focus group I (FG I) included young people aged $15-17$ years $(n=4 ; 2$ females and 2 males); Focus group II (FG II) included 1 male and 1 female, aged between 11 and 13 years. Reasons for service referral were school- and family-related $(n=3)$, internalising problems $(n=2)$ and externalising problems $(n=2)$. Participants had attended between 7 and 30 sessions, involving a mix of individual, family and school-based treatment. Four cases were closed, and three were ongoing.

Phase II. Phase II inclusion criteria were extended to include (1) youth attending a community firstline clinic and (2) youth who required language assistance from a Swedish interpreter. All other inclusion criteria remained the same. In all, 587 children and adolescents were invited to participate. A total of 348 service users (mean age $=14.77$ years, standard deviation $(S D)=2.37,67.7 \%$ female) responded to the survey (59.3\%), including 106 young people who provided 123 comments on what would have improved their appointments (mean age $=14.56$ years, $S D=2.50,80.2 \%$ female). 
There was no difference in mean age between those who gave comments and those who did not $(t=.77, p<.05)$, although females were overrepresented among those providing comments $(Z=2.47, p<.05)$. Participants came from 14 different clinics situated in nine different regions across Sweden.

\section{Measures}

Phase I interviews. A semi-structured topic guide included open-ended questions and interactive exercises to explore children's and adolescents' experiences of mental health care (available from the authors on request). Examples included, 'How many marks out of 5 would you give the appointments? Why such a high/low score? Is there anyone who agrees/disagrees', 'What have been the best/worst aspects of the appointments?', 'Write down three things that you think are most important about the appointments, it can be "good" or "bad" things, then we will discuss what you saw as important'.

Phase II surveys. Young people answered the free-text question, 'Please, give two suggestions on what would have made your visits at the clinic better for you?' This item was nested within a longer service experience questionnaire with 26 forced-choice items. Only the qualitative data are reported here. Two versions of the questionnaire were distributed, one for younger children (10-14 years) and one for older adolescents (15-18 years). The two versions were identical, except the former contained slightly simplified wording and included illustrations reflecting the meaning of each item.

\section{Procedure}

Focus groups. Clinicians presented potential participants with oral and written information about the study. Young people who expressed an interest and completed a contact form were then followed up in a telephone call from a researcher. An initial discussion emphasised that participation was voluntary, they could choose to withdraw at any point, and interviews would be recorded and saved as audio files. Furthermore, they were told that results would be presented in such a way so that no individual could be identified, and they were free to speak as little or much as they wished. Room was given for questions from participants. Young people who wished to continue with the study were asked to sign a written consent form. For those under 15 years of age, parental consent was also sought. Participants were reimbursed for their time with a movie ticket and received travel expenses.

Consenting participants were divided into two separate focus groups according to age (11-13 and 15-17years). Each focus group met once in a clinic conference room in the early evening. Focus groups were led by the first author (S.P.) who acted as interviewer; a certified psychologist assisted and was present as a resource. At the outset, participants were asked to fill out a form asking about age, sex, number of appointments, and reason for/type of treatment.

Interviews aimed to generate a discussion about participants' experiences of the service. The facilitator had a warm and encouraging style and was alert to potential overdisclosure (e.g. sharing details of why they had contact with the clinic). Open and clarifying follow-up questions were used. Participants were also asked if any important questions or discussion points had been left out at the end of each focus group. FG I lasted 70 minutes and FG II took 32 minutes.

Validation interviews. Focus group participants were invited to a subsequent round of validation interviews (Graneheim \& Lundman, 2004; Long \& Johnson, 2000). The relatively small number 
of respondents ( $n=3$, aged 15-17 years, one female) meant that individual interviews were carried out instead of using a group format. Respondents were presented with a booklet in which suggested thematic categories were presented together with illustrative quotes. Participants were asked to discuss and rate (important, somewhat important and not important) the relative importance of the categories in determining the subjective experience of an appointment. Young people were also asked to consider whether any important determinants of service experience were missing from the initial set of thematic categories. The interviewer (S.P.) went through the booklet presenting each category to the participants. Interviews were audio-recorded and lasted 30-60 minutes.

Survey data. The survey was distributed by an administrator, together with an envelope containing written information about the research. Participants were told that participation was voluntary and that they could fill out the survey in the waiting area or mail it (using a prepaid envelope). Written consent from parents was required for participants aged 10-14 years; participants aged 15-18 years gave consent by answering the questionnaire.

\section{Analysis}

Focus groups and validation interviews. Focus group interviews were transcribed verbatim in Swedish and subjected to qualitative content analysis following Graneheim and Lundman's (2004) recommendations. First, a transcription of each focus group interview was read to reach an initial understanding of the material. Second, participants' experiences of their appointments were extracted; meaning units were identified and abstracted into labelled codes. Third, codes were examined for similarities and differences, as they were sorted into subcategories and categories, relating the parts of the text to the whole. The initial analysis was performed by S.P. and refined through iterative consultations with co-authors.

Validation interviews. Audio recordings from the validation interviews were reviewed by S.P. alongside notes about the importance attached to categories in the validation stage. None of the categories were judged to be irrelevant based on statements or ratings, although there were indications that younger children might place relatively greater importance on creative and enjoyable session activities. However, no changes were indicated to the thematic categories.

Surveys. Free-text comments from the surveys were subjected to qualitative content analysis (Graneheim \& Lundman, 2004). Comments were entered into a spreadsheet and condensed into meaning units which were then assigned codes. These codes were abstracted into subcategories and categories describing different areas for service improvement. The analysis was performed by S.P.; results were continuously discussed with a colleague not involved in the project.

In the first instance, focus group and survey data were analysed separately. The categories from each data source were then combined and organised into three overarching categories of service experience. In integrating the two data sources, the focus group data provided depth and the improvement suggestions provided breadth.

\section{Results}

The focus group data revealed four categories of service experience ('Accessibility', 'Environment', 'Ease of communication' and 'Outcomes') while the survey data revealed four areas of improvement ('Improved accessibility', 'A nicer care environment', 'Increased possibilities for being heard and seen' and 'Help in understanding and dealing with the problem'). These eight categories were 
Table I. The three main themes derived from the analysis of focus group- and survey data.

\begin{tabular}{llll}
\hline & Accessibility & Being heard and seen & Usefulness of sessions \\
\hline $\begin{array}{l}\text { Focus } \\
\text { groups }\end{array}$ & Accessibility & Ease of communication & Outcomes \\
Survey & $\begin{array}{l}\text { Environment } \\
\text { Improved accessibility } \\
\text { Nicer care environment }\end{array}$ & $\begin{array}{l}\text { Possibilities for being } \\
\text { heard and seen }\end{array}$ & $\begin{array}{l}\text { Understanding and } \\
\text { dealing with the problem }\end{array}$ \\
\hline
\end{tabular}

combined into three overarching themes: 'Accessibility', 'Being heard and seen' and 'Usefulness of sessions', presented below (quotations from focus groups: FG I = 15-17 years, FG II =11-13 years; $\mathrm{Q}=$ questionnaire data). In Table 1, the categories from respective data source are presented.

\section{Accessibility}

Children and adolescents discussed and made suggestions concerning how services could make them feel more welcome and relaxed, as well as pointing out problems related to appointment times. This is described in the three subcategories 'Care environment', 'Appointment times' and 'Travel'.

Care environment. Participants discussed the importance of both interpersonal and material factors in creating an environment where they felt welcome, relaxed and safe: '. . you should feel safe when coming here ... if they had not been kind I would not feel safe to come here and talk' (FG II). Young people expressed that it was the responsibility of all service staff - not only clinicians - to make service users feel valued and comfortable. The physical surroundings also played an important role, such that more informal and homely settings were favoured over sterile hospital environments. Suggestions for creating a more attractive and informal care setting included brighter and happier colours, as well as more comfortable and cosier furnishing in the waiting area and session rooms: 'Less feeling of institution, that is, more personal furnishing and decoration' (Q: Boy, age 16). Moreover, children and adolescents wanted to be able to influence the milieu in the session room, for example deciding whether to have lights on or off, and whether to have some music in the background. Refreshments were suggested as another way of making young people feel welcome: 'Offer refreshments because: feels nicer' (Q: Girl, age 12). Some comments about being offered refreshments were ironic in a way that can be interpreted as 'yeah, like that would happen': 'Refreshments, ha ha ha' (Q: Girl, age 11); 'Be offered tea, haha' (Q: Girl, age 16).

Appointment times. The focus groups revealed dissatisfaction with appointments being scheduled at inconvenient times, starting too late due to the therapist being off-schedule and finishing earlier than planned. Relatedly, it was pointed out that it could be very stressful knowing that you were missing out on something important in school while being at a clinic session: 'Earlier I always had appointments in the morning and that was not good, I had school then, I thought it was rather bad '(FG II).

Consistent with this theme, survey suggestions included more flexible appointment times that would allow for attendance outside school hours: 'Better appointment times, [to avoid] missing out on much in school' (Q: Girl, age 17). Longer visits were also suggested to avoid the feeling of being rushed: 'Possibility for longer visits, without stress' (Q: Girl, age 17). Other suggestions included more punctual behaviour by clinicians, the possibility for drop-in visits and availability of telephone consultations. 
Travel. Focus groups highlighted further concerns about the process of travelling to the clinic (e.g. by public transport, driving with parents). Some mentioned that they did not live in the city close to the clinic, leading to difficulties with transportation: 'I live in the countryside . . . with not so many available buses' (FG I).

\section{Being heard and seen}

This theme contains suggestions that might improve children's and adolescents' opportunities for sharing their problems, and feeling respected. The 'Ease of communication in sessions' subcategory relates to pacing and openness in therapeutic encounters. The other two subcategories ('Match and vary session activities' and 'Show interest in the whole person') describe a need for developmentally appropriate methods of communication, and a wish for being seen as a whole person, with an identity that extends beyond the presenting problem(s).

Ease of communication in sessions. During sessions, young people expected to be able to disclose 'What is it that makes it [the session] good?' . . 'you can talk . . about everything' (FG II). The therapist needs to be relatable, engaging and capable of putting young people at ease: '. . . it's important to feel that you can talk to the person you meet' (FG I). An open session climate was preferred, where a young person is able to express what $\mathrm{s} / \mathrm{he}$ wants to say without interruption, coercion or fear of being judged: 'I think it is important to respect one another and let all say what they want to' (FG I). Sessions should be paced in such a way that young people do not feel rushed into disclosing personal issues too quickly. General suggestions were offered about gradually building trust and mutual understanding: 'You can build a relation before starting to talk' (Q: Girl, age 17); 'To get to know each other at the same time' (Q: Girl, age 11). The use of humour was also identified as potentially helpful in the process of building an open and relaxed rapport.

A constrained session climate was indicated by feeling pressure to disclose. Different types of pressure were discussed: feeling pressured to 'say the right things' or reveal too much, and also fear of giving the wrong answer: 'You should not feel pressured to say the correct things . . and no pressure to say more than you want to' (FG I). There was agreement in both focus groups that parents and other adults have an impact on the conversation in terms of what is possible to bring up, compared to when only the clinician is in the room: 'Some things you don't want to talk about... because it is a bit private, but parents want to'; 'I think it's better to be alone [with the clinician] because I talk better when I'm alone' (FG II). This was also brought up among the improvement suggestions: 'Do not begin the conversation with [the problem], in front of my parents' (Q: Boy, age 16). Further concerns were raised about being interrupted or overruled by competing voices when other adults are involved in sessions. Silences can also be uncomfortable: some younger children referred to awkward feelings when the therapist did not speak, and they did not know what to say or what was expected from them in response.

Match and vary session activities. Young people, especially those aged under 14 years, made suggestions about nonverbal methods for communicating their thoughts and feelings: 'To write and draw how it feels' (Q: Girl, age 11). For some young people, verbal communication can be facilitated by physical outlets: 'If I could do something while talking' (Q: Girl, age 13); 'Occupation for my hands, helps me to focus' (Q: Girl, age 15). Variation, interactivity and fun in session activities were also mentioned: 'A 5 or 10 minute break, maybe do something fun during the break' (Q: Girl, age 12); 'More variation, for example a game of some kind' (Q: Girl, age 15). This may be more engaging and acceptable than more formal written tasks, which can be stressful for younger children in particular. However, careful consideration of an individual's interests and capacities is necessary when 
selecting session activities: even for the youngest participants in our sample, concerns were raised about activities being pitched below their developmental level, and appearing as 'childish'.

Show interest in the whole person. Children and adolescents described negative experiences where clinicians had not really paid attention or listened: 'It happened that the clinician had forgotten what I had said . . . maybe he should take notes/prepare himself better' (Q: Girl, age 16). In focus groups, perceptions of the clinician not really listening or not taking the young person seriously were related to feeling like an object, like someone who should be 'fixed': 'I believe it makes you feel more as a case .. . could be hurtful ... if you are not so well treated' (FG I). Preferential attention towards parents also made the young people feel as if clinicians did not believe in what they were saying: 'If, for example, mother is present it might be that he [the clinician] agree with mother . . . but it shouldn't be like that'(FG II).

Among the improvement suggestions, participants expressed the importance of feeling that the clinician was genuinely interested in them as a person, beyond the nature of their problem(s): 'If he hadn't focused on just one thing [the problem], but also had showed concern for how I was feeling in general' (Q: Girl, age 16); 'Show understanding for why one feels as one does, not making one feel as being less intelligent' (Q: Girl, age 17). In focus groups, children and adolescents expressed a wish for being asked what they would like to bring up at sessions, which was related to having opportunities to talk about other problems besides the 'big issue': '. . . to get the help you need at the moment ... what worries you the most' (FG I). This was also brought up as an area for improvement: 'More of what I wanted to discuss' (Q: Girl, age 16).

\section{Usefulness of sessions}

This theme describes young people's wishes for receiving relevant and effective treatment that impacts on presenting problems, and respects their agency in attending sessions.

Help in dealing with the problem. Focus group participants discussed the importance of clinicians giving good advice that actually works, knowing what to say, and more generally having the capacity to make young people feel better. Treatment should be focused and relevant: ' . . it is important to receive the help you need' (FG I). Suggestions were made for advice being more understandable, with practical tools 'that were more concrete' (Q: Girl, age 18). Furthermore, there was a wish for an overall clearer structure regarding treatment goals: 'Better structure concerning how the treatment is supposed to help me' (Q: Girl, age 17); 'Given me clearer guidelines and help for my problems' (Q: Girl, age 17).

Perceived agency. Feeling pressure from others such as parents and school professionals to attend treatment sessions was brought up in both focus groups: 'I went not because I wanted it, it was the school and others who wanted it . . so I went because they asked me . . went although I didn't want to' (FG I). At times, young people felt as if it was their parents who wanted to talk about the problem or who had a problem. Under these circumstances, it was expressed that attending appointments felt rather unnecessary: 'It probably did nothing for me, I mostly went because mother wanted me to' (FG II).

\section{Discussion}

This study explored young service users' experiences of mental health care in Sweden. The analysis revealed three service priorities: (1) accesible services where young people are offered timely 
appointmentsin a convenient and welcoming setting, (2) opportunities to communicate openly and participate in developmentally appropriate session activities, where young people feel engaged, understood and recognised as a whole person and (3) meaningful and effective treatment, which is structured, practical, and responsive to young people's priorities and motivations in help-seeking.

The results reaffirm earlier international findings regarding the importance that children and adolescents attach to their relationship with the therapist (Biering, 2010; Lavis \& Hewson, 2011) which is part of most service experience measures (e.g. Ayton, Mooney, Sillifant, Powls, \& Rasool, 2007; Brown, Ford, Deighton, \& Wolpert, 2014; Day et al., 2011), as well as satisfaction with the care environment (e.g. Brown et al., 2014). The focus group discussions as well as the written improvement suggestions make clear that children and adolescents value sessions where the clinician actively listens and validates their point of view, freeing the young person to speak openly. As revealed in other studies (Day et al., 2006; Strickland-Clark et al., 2000), the (over)involvement of parents in sessions can pose significant challenges to the development of this trusting therapeutic relationship. Clinicians should therefore place an overt emphasis on balancing the views of parents and young people in the process of care, while allowing opportunities for young people to meet privately if desired. This is especially important since young people may feel compelled by parents and school professionals to engage in treatment (Ivert et al., 2011). Those who are in treatment because of external pressure may be at greater risk for drop-out (de Haan et al., 2013), having less favourable outcomes of treatment (Ryan \& Deci, 2008), and generally being less satisfied with services (Garland, Aarons, Saltzman, \& Kruse, 2000).

Sensitive pacing of sessions is also important, so that young people do not feel rushed into overdisclosure (Hartzell et al., 2009). A gradual process of getting to know each other also affirms a young person's individual identity so that it encapsulates more than their presenting problems. Other recommendations were made about the methods of communication, with indications that younger adolescents preferred creative, non-verbal ways of conveying their feelings and problems.

Other research studies have shown that physical surroundings may be relatively less influential than in-session activities and interpersonal factors in determining young people's overall perception of service quality (Day et al., 2011). Nevertheless, as revealed in the present study, availability of comfortable furnishings, warm decor and refreshments may have a positive bearing on initial perceptions of a service as being safe and welcoming. Young people also suggested having background music in waiting rooms, which has been found to have calming effects in other health-care contexts (Brittain \& Jones, 2009; Cooke, Chaboyer, \& Hiratos, 2005). This is important since young people can experience high levels of apprehension and anxiety about attending appointments in CAMHS, especially at the outset of their care (Gulliver, Griffiths, \& Christensen, 2010). Other research has shown that such anticipatory anxiety may be related to a lack of knowledge about what to expect when arriving at clinics for the first time (Bone, O’Reilly, Karim, \& Vostanis, 2014). On this last point, our participants pointed out that they wanted more information regarding the structure and potential benefits of treatment. They also favoured clear and concrete advice about how to improve and cope with their problems. Attending to these preferences must be a priority in order to overcome ambivalence about session attendance, and enhance treatment participation and outcomes.

Getting to appointments can also present difficulties - even when clinics are located in the community. Using public transportation can be cumbersome, and it was not always possible to get a lift with parents. Furthermore, appointment times that are in conflict with school hours were seen as problematic, as brought up by young people in an earlier study (Day et al., 2006). Consequently, young people sought to have more influence over the choice of appointment times. Moreover, young people expressed frustration over sessions not starting and finishing on time. Meeting young people's wishes for accessible care is important, not least as mental health and school results are reciprocally related (Gustafsson et al., 2010). 


\section{Strengths and limitations}

The study has several strengths. First, specific recommendations were obtained for mental healthcare improvements based on young people's own suggestions. This offers an ecologically rich source of data to inform service development. Second, external validity was further enhanced by recruiting a sample from 'real-world' clinics representing a range of services and regions. Third, internal validity was strengthened by triangulating data from focus groups and surveys. The resulting thematic analysis was subsequently verified in consultation with a subgroup of participants. Despite these methodological strengths, we cannot rule out the possibility of sampling bias related to (1) the smaller than intended focus groups, and (2) lack of representativeness among survey respondents. In particular, young people with specific difficulties in expressing themselves in writing might be underrepresented in our sample. On the other hand, we found a strong degree of overlap between the themes that emerged from the focus groups and anonymised surveys.

\section{Conclusion}

This study examined users' experiences of CAMHS in Sweden and found substantial correspondence with priorities identified in other international research on youth service experience. In addition, this study provides services in Sweden and elsewhere with a range of specific practice recommendations from children and adolescents. These offer potentially viable improvements that could be implemented and evaluated for impact. In a related project, we will validate a quantitative measure of service experience for use in Sweden. The purpose is to develop a reliable tool that can support service improvement efforts of the type considered here. This programme of research should enable services to move beyond the rhetoric of 'youth-friendly' care, by informing systematic and measurable improvements that capture the real priorities and preferences of young people.

\section{Acknowledgements}

We would like to acknowledge Fredrik Lindencrona and Sofie Sjöborg, the Swedish Association of Local Authorities and Regions, for support and collaboration.

\section{Funding}

The author(s) disclosed receipt of the following financial support for the research, authorship, and/or publication of this article:This work was financially supported by the National Board of Health and Welfare in Sweden.

\section{References}

Ayton, A. K., Mooney, M. P., Sillifant, K., Powls, J., \& Rasool, H. (2007). The development of the child and adolescent versions of the Verona Satisfaction Scale (CAMHSSS). Social Psychiatry and Psychiatric Epidemiology, 42, 892-901.

Biering, P. (2010). Child and adolescent experience of and satisfaction with psychiatric care: A critical review of the research literature. Journal of Psychiatric and Mental Health Nursing, 17, 65-72.

Bone, C., O'Reilly, M., Karim, K., \& Vostanis, P. (2014). 'They're not witches . . ' Young children and their parents' perceptions and experiences of Child and Adolescent Mental Health Services. Child: Care, Health, and Development, 41, 450-458.

Bor, W., Dean, A. J., Najman, J., \& Hayatbakhsh, R. (2014). Are child and adolescent mental health problems increasing in the 21 st century? A systematic review. Australian and New Zeeland Journal of Psychiatry, $48,606-616$. 
Brittain, D., \& Jones, M. (2009). Music in the waiting room. The British Journal of General Practice, 59, 613-614.

Brown, A., Ford, T., Deighton, J., \& Wolpert, M. (2014). Satisfaction in child and adolescent mental health services: Translating users' feedback into measurement. Administration and Policy in Mental Health and Mental Health Services Research, 41, 434-446.

Buston, K. (2002). Adolescents with mental health problems: What do they say about health services. Journal of Adolescence, 25, 231-242.

Cooke, M., Chaboyer, W., \& Hiratos, M. A. (2005). Music and its effect on anxiety in short waiting periods: A critical appraisal. Journal of Clinical Nursing, 14, 145-155.

Day, C., Carey, M., \& Surgenor, T. (2006). Children's key concerns: Piloting a qualitative approach to understanding their experience of mental health care. Clinical Child Psychology and Psychiatry, 11, 139-155.

Day, C., Michelson, D., \& Hassan, I. (2011). Child and adolescent service experience (ChASE): Measuring service quality and therapeutic process. British Journal of Clinical Psychology, 50, 452-464.

de Haan, A. M., Boon, A. E., de Jong, J. T., Hoeve, M., \& Vermieren, R. R. (2013). A meta-analytic review on treatment dropout in child and adolescent outpatient mental health care. Clinical Psychology Review, $33,698-711$.

Freake, H., Barley, V., \& Kent, G. (2007). Adolescents' views of helping professionals: A review of the literature. Journal of Adolescence, 30, 639-653.

Garland, A. F., Aarons, G. A., Saltzman, M. D., \& Kruse, M. I. (2000). Correlates of adolescents' satisfaction with mental health services. Mental Health Services Research, 2, 127-139.

Garland, A. F., Haine, R. A., \& Lewczyk Boxmeyer, C. (2007). Determinates of youth and parent satisfaction in usual care psychotherapy. Evaluation and Program Planning, 30, 45-54.

Garland, A. F., Lewczyk Boxmeyer, C. M., Gabayan, E. N., \& Hawley, K. M. (2004). Multiple stakeholder agreement on desired outcomes for adolescents' mental health services. Psychiatric Services, 55, 671676.

Graneheim, U. H., \& Lundman, B. (2004). Qualitative content analysis in nursing research: Concepts, procedures and measures to achieve trustworthiness. Nurse Education Today, 24, 105-112.

Gulliver, A., Griffiths, K. M., \& Christensen, H. (2010). Perceived barriers and facilitators to mental healthseeking in young people: A systematic review. BMC Psychiatry, 10, 113.

Gustafsson, J.-E., Allodi, M. W., Alin Åkerman, B., Eriksson, C., Eriksson, L., Fischbein, S., . . Persson, R. S. (2010). School, learning and mental health: A systematic review. Stockholm: The Royal Swedish Academy of Sciences, The Health Committee.

Harper, B., Dickson, J. M., \& Bramwell, R. (2014). Experiences of young people in a 16-18 mental health service. Child and Adolescent Mental Health, 19, 90-96.

Hartzell, M., Seikkula, J., \& von Knorring, A. (2009). What children feel about their first encounter with child and adolescent psychiatry. Contemporary Family Therapy, 31, 177-192.

Ivert, A. K., Svensson, R., Adler, H., Levander, S., Rydelius, P. A., \& Levander, M. T. (2011). Pathways to child and adolescent psychiatric clinics: A multilevel study of the significance of ethnicity and neighbourhood social characteristics on source of referral. Child and Adolescent Psychiatry and Mental Health, 5, 1-12.

Lavis, P., \& Hewson, L. (2011). How many times do we have to tell you? A briefing from the National Advisory Council about what young people think about mental health and mental health services. London: National Advisory Council.

Long, T., \& Johnson, M. (2000). Rigour, reliability and validity in qualitative research. Clinical Effectiveness in Nursing, 4, 30-37.

McCann, T. V., \& Lubman, D. I. (2012). Young people with depression and their satisfaction with the quality of care they receive from a primary care youth mental health service: A qualitative study. Journal of Clinical Nursing, 21, 2179-2187.

McLeod, B. D. (2011). Relation of the alliance with outcomes in youth psychotherapy: A meta-analysis. Clinical Psychology Review, 32, 603-616.

National Board of Health and Welfare. (2013). Barns och ungas hälsa, vård och omsorg [Children's and young peoples' healthcare]. Stockholm, Sweden: Socialstyrelsen (in Swedish). 
Ryan, R. M., \& Deci, E. L. (2008). A self-determination theory approach to psychotherapy: The motivational basis for effective change. Canadian Psychology, 49, 186-193.

Strickland-Clark, L., Campbell, D., \& Dallos, R. (2000). Children's and adolescents' views on family therapy. Journal of Family Therapy, 22, 324-341.

World Health Organization. (2012). Making health services adolescent friendly: Developing national quality standards for adolescent friendly health services. Geneva, Switzerland: World Health Organization, Department of Maternal Newborn Child and Adolescent Health.

Yeh, M., \& Weisz, J. R. (2001). Why are we here at the clinic? Parent-child (dis)agreement on referral problems at outpatient treatment entry. Journal of Consulting and Clinical Psychology, 69, 1018-1025.

Zachrisson, H. D., Rödje, K., \& Mykletun, A. (2006). Utilization of health services in relation to mental health problems in adolescents: A population study. BMC Public Health, 6, 34.

\section{Author biographies}

Stefan Persson, PhD, is a Senior Lecturer in Psychology at Karlstad University, Sweden. His research concerns quality in mental health care from children's and adolescents' perspectives, and how their experiences can be used in service development.

Curt Hagquist is Professor of Public Health, Director and founder of the Centre for Research on Child and Adolescent Mental Health at Karlstad University, Sweden. He is a trained social worker and gained his PhD in Social work at the University of Gothenburg. His research fields include adolescent mental health, epidemiology, Rasch Measurement Theory, and social determinants of health.

Daniel Michelson is an academic clinical psychologist based at King's College London and the University of Oxford. His research focuses on the development and evaluation of innovative mental health service models for youth and families, with a particular emphasis on socially disadvantaged and 'hard-to-reach' groups. 